\title{
A Technologists Viewpoint
}

\author{
Liz Clarke
}

\subsection{Training}

In the early days training for technicians varied throughout the UK. In the 1980s in the Midlands for example there was a West Midlands Regional Health Authority training scheme in Medical Physics and Physiological Measurement. This 2 year course required "O" Level (GCSE) qualifications to include scientific subjects. It consisted of block release study for an Ordinary National Certificate (ONC) alongside hospital placements in Nuclear Medicine, Radiotherapy (Planning and Mould room), Radiation Protection, Audiology, Neurophysiology, Cardiology, and Renal units. Trainees then decided in which area they would like to specialise and could go on to pursue a Higher National Certificate (HNC) in this area. Again this could be a Regional training scheme or study in post.

The training of technologists has changed over the years to part time BSc course's with minimum requirement of " $A$ " Level qualification which is run by a small number of universities. Additional modules allow for training on reporting, cardiac stressing and CT. This reflects the increased complexity of equipment and procedures now in place in departments and indeed the increased knowledge required to fulfil the role.

Specific courses were also developed to concentrate on the educational needs for radiographers in the use of radiopharmaceuticals in imaging. Radiography training was originally a 2 year diploma course and then progressed to a 3 year degree course. There is very little nuclear medicine training at undergraduate level and it has been necessary to continue to postgraduate training. Some staff have relied on in-house training with no formal qualification. Formal qualification originally was the Diploma in Nuclear Medicine of the College of Radiographers which came into existence around 1972,

\footnotetext{
L. Clarke

European Applications, GE Healthcare Ltd, The Grove Centre,

White Lion Road, Amersham HP7 9LL, UK 
prior to that it was a local course run by the Royal Marsden Hospital in Sutton. This included therapy and in-vitro work. This was phased out around 1983 by the College of Radiographers and replaced with the Diploma in Radionuclide Imaging (DRI) which was also open to technicians and was delivered in association with the Schools of Radiography. At present some universities organise Masters of Science degrees in nuclear medicine, with options to step off at post graduate diploma and post graduate certificate levels depending upon what level of education and training is needed.

The Register of Clinical Technologists (RCT) was established in 2000 with the aim of advocating statutory, professional regulation for Clinical Technologists (www.therct.org.uk). Clinical Physics Technologists are included on this register that includes: Scope of practice, Code of conduct and Continuing Professional Development. This register has recently received accreditation from the Professional Standards Authority and continues to strive for recognition as statutory regulation.

\subsection{The Role}

When radio-isotope departments were developed in the early 1950s most studies on patients involved laboratory based techniques using blood samples etc. Usually run by physicists who often made their own equipment with the investigations carried out by physics technicians.

As time and technology progressed, imaging became more important and while laboratory studies continued, physics technicians became more involved with patients. Early imaging was performed on a rectilinear scanner which produced very noisy images often on large paper prints. Things improved when single headed gamma cameras were introduced although achieving the correct exposure initially could be a problem. Large film cassettes were transported to communal dark rooms shared with Radiology to develop the films that were reliant on intensity and f-stop settings! Getting these wrong meant the repeat of an entire study. The advent of the Gamma Camera made life a lot easier. In Fig. 4.1 Neil Smith is imaging a patient with a Scintronix gamma camera in 1982. The images were still being recorded on x-ray film.

At this time although the jobs performed by both technicians and radiographers working in Nuclear Medicine departments were generally identical, working conditions such as weekly hours and annual leave entitlements were different. Today, following Agenda for Change, there is less difference between the two groups.

Cross sectional imaging started with SPECT and progressed to multimodality SPECT/ CT for attenuation correction, PET/CT and now PET/MRI. With the further introduction of multi-slice diagnostic CT scanners this itself raises the question of CT training for non-radiographers and also radiographers who have not had formal CT training. The introduction of University modules has helped to address this issue.

Every department will differ but generally the daily role has evolved to include radio pharmacy, dispensing, patient injection and supervision of cardiac stress sessions. The introduction of hybrid technologies has increased the complexity of acquiring and processing the studies leading to a demand for the staff to upskill. Some have undertaken further training to enable them to report and others are also business managers leading the technical side of their departments. 


\subsection{Research}

Radiographers and technicians are often involved in strong research programs which have them taking a lead in the study itself; presenting and publishing the results. Dudley Road Hospital, Birmingham under the direction of Dr Keith Harding in particular led the way with its technicians presenting the results of their studies at the BNMS and International meetings, over 60 publications from their technologist team between 1985 and 2002 (Fig. 4.2). Topics covered a range of Nuclear Medicine procedures and Radiation Protection [1-4]. Nationally this practitioner involvement and enthusiasm has led to the introduction of dedicated Technologist sessions at

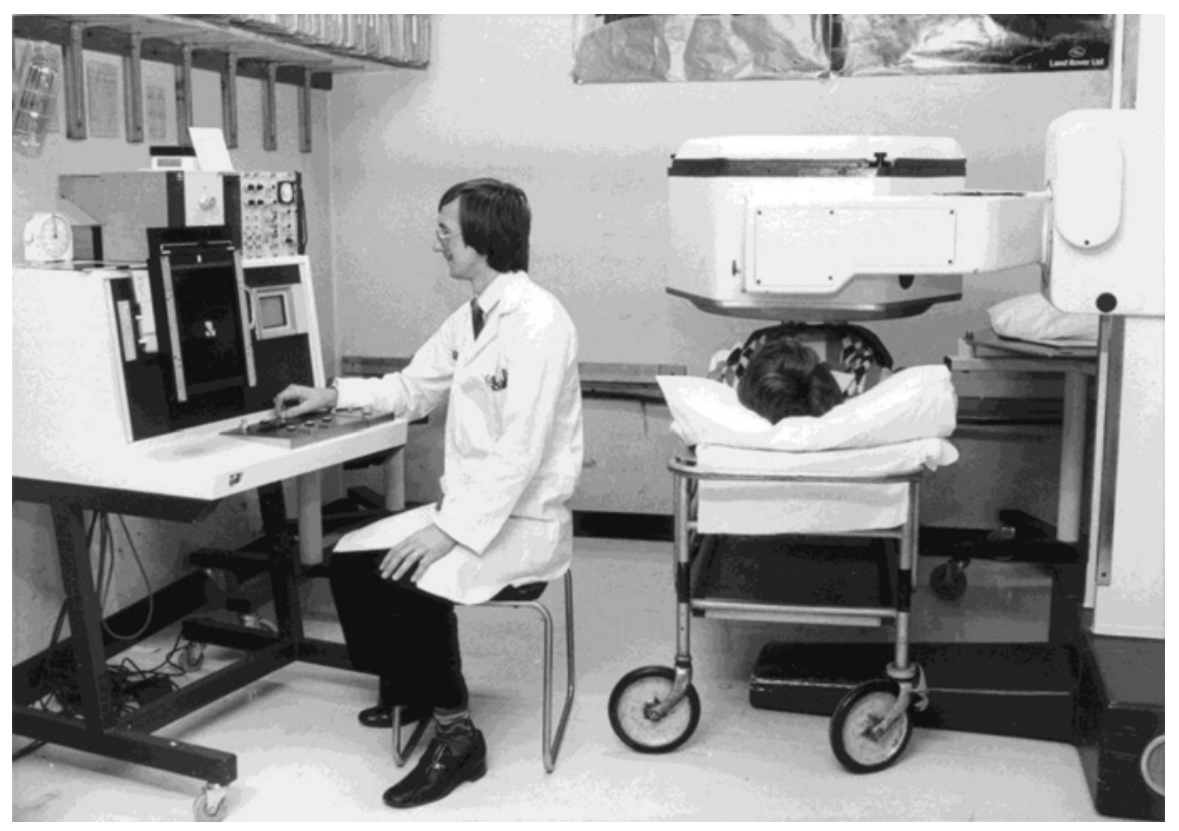

Fig. 4.1 Imaging on a Scintronix Gamma Camera in 1982

Fig. 4.2 Dr Keith Harding

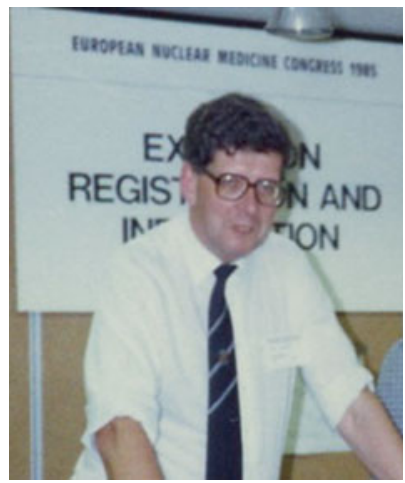




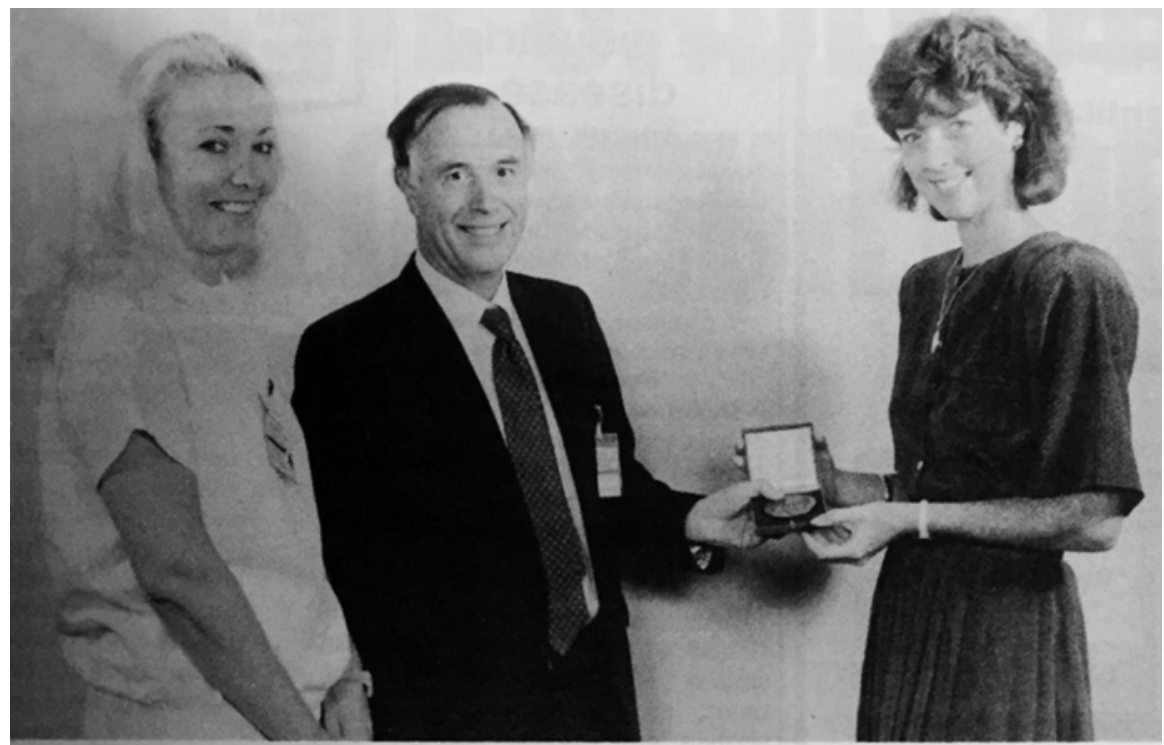

Pictured from left are Audrey Painter, Product Specialist (nuclear medicine) with Mallinckrodt Medical England, Dr Ralph McCready, chairman of the Task Group on Continuing Education, European Association of Nuclear Medicine, and prizewinner Liz Clarke.

Fig. 4.3 The first Mallinckrodt award for the best Technologist presentation at the EANM is presented to Liz Clarke (Courtesy Rad Magazine)

national and international meetings with proffered papers from across the UK and awards for best presentations. The first Mallinckrodt European Technologist award (META) for the best technologist presentation at the EANM was won by a UK technologist Liz Clarke (the senior technician at Dudley Road Hospital) Fig. 4.3 [5].

Another example of work by practitioners which in this case also included nurses was the publication of guidelines in Nuclear Medicine Communications for training for non-medical supervision of cardiac stress tests [6].

\subsection{BNMS and Professional Development}

At the EANM Congress 1989 in Strasbourg technicians and radiographers were refused entry into some clinical sessions. This led to an inaugural meeting of the first British Nuclear Medicine Society Technologist group which took place on the steps of the Congress centre. On their return to the UK the group began establishing itself in earnest with Caroline Townsend (Consultant Radiographer, UCH) as its first Chairperson and Liz Clarke as Secretary. A council consisting of a mix of technicians and radiographers from across UK and Ireland decided after much debate on the name of Technologists Group and a constitution was written. The group was 


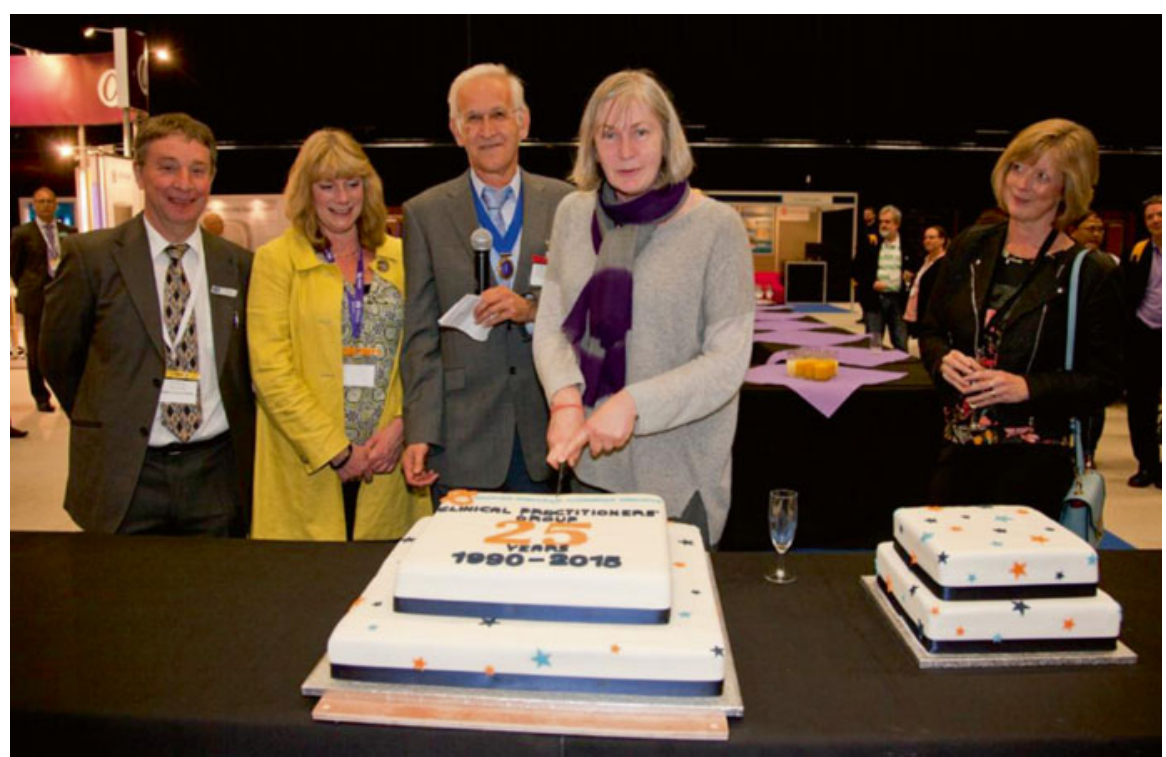

Fig. 4.4 The cake cutting ceremony at the celebration of the 25th anniversary of the BNMS clinical practitioner group. From left to right Ashby GB's Phil Facy, Mallinckrodt's Vicki Parkin, BNMS President Alp Notghi, Caroline Townsend (UCHL) and GE Healthcare's Liz Clarke (Courtesy Rad Magazine)

officially acknowledged as part of the BNMS in 1990. The EANM took our lead and established a European Technologist group established with the involvement of the UK a couple of years later. The aim of these groups was to unite all those working in all aspects of Nuclear Medicine regardless of their professional routes.

The name has recently changed again to Clinical Practitioners to better reflect the large variety and extent of expertise within the group. 2015 saw the celebration of 25 years of the group and a further change with the inclusion of nurses working within the specialty. It has been suggested that this may lead to a further change of name in 2016 (Fig. 4.4).

\subsection{The Future}

Radiographers and technologists continue to play a vital role in Nuclear Medicine.

They will have to ensure to increase their range of expertise to match the new technology and should embrace their opportunities to participate in research and continue their professional development.

Reporting of studies, research leading to $\mathrm{PhDs}$, therapeutic administrations and Clinical Practitioner led patient clinics are just some of the extended advanced practice roles that are been undertaken. 
Open Access This chapter is distributed under the terms of the Creative Commons AttributionNoncommercial 2.5 License (http://creativecommons.org/licenses/by-nc/2.5/) which permits any noncommercial use, distribution, and reproduction in any medium, provided the original author(s) and source are credited.

The images or other third party material in this chapter are included in the work's Creative Commons license, unless indicated otherwise in the credit line; if such material is not included in the work's Creative Commons license and the respective action is not permitted by statutory regulation, users will need to obtain permission from the license holder to duplicate, adapt or reproduce the material.

\section{References}

1. Sherwin S, Donovan IA, Hesslewood SR, Sorgi M, Alexander-Williams J, Harding LK. DiethylHIDA concentration in bile, blood and urine. Nucl Med Commun. 1982;3:102 (Abstract).

2. Childs PO, Mostafa AB, Causer DA. A quantitative evaluation of breathing systems used with Kr-81m generators. J Nucl Med. 1983;24:157-9.

3. Allen C, De Bolla A, Tulley NJ, Harding LK, Barnes AD, Heath DA. The importance of the substraction technique in detecting parathyroid adenoma? Eur J Nucl Med. 1985;11:A47 (Abstract A251).

4. Clarke EA, Notghi A, Harding LK. Improving myocardial perfusion protocols: results of a UK survey. Nucl Med Commun. 2002;23:414 (Poster).

5. Clarke EA. Nuclear medicine: a safe occupation during pregnancy? Nucl Med Commun. 1989;10:260 (Abstract).

6. Jones I, Latus K, Bartle L, Gardner M, Parkin V. Clinical competence in myocardial perfusion scintigraphic stress testing: general guidelines and assessment. Nucl Med Commun. 2007; 28(7):575-82. 


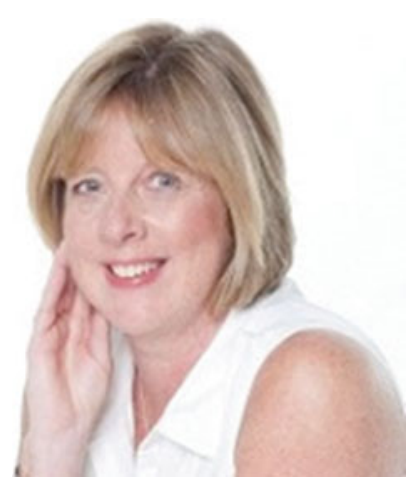

Liz Clarke European Applications Specialist Team Leader, GE Healthcare Life Sciences.

I worked in the Nuclear Medicine Department at Birmingham City Hospital (formerly Dudley Road) for 21 years. During that time I was involved in many research areas and presented many times at the BNMS and EANM. I was lucky enough to be awarded the Best Technologist paper on many occasions and was twice recipient of the Mallinckrodt award at the EANM. Much of this work was Radiation Protection orientated especially in regard to working during pregnancy and new imaging techniques in SLN imaging. Dr Keith Harding was my mentor whom I both feared and greatly respected simultaneously! I certainly owe all of my presenting skills to his superior knowledge and encouragement.

After great deliberation I left the NHS in 2002 to join Amersham Health (now GE Healthcare) to continue my career as an Applications Specialist. Although very different it has enabled me to further develop my expertise and continue to work within the Nuclear Medicine community, many of whom are now my friends as well as working colleagues. 